\title{
ÉTUDE COMPAREE DES RÉSERVES VITELLINES ET DE LA DURÉE DE LA PHASE DE GRAND ACGROISSEMENT DE L'OVOCYTE CHEZ LA POULE DOMESTIQUE. INFLUENCE DE L'AGE DE L'ANIMAL ET DE LA LONGUEUR DE LA SÉRIE
}

PAR

\section{LACASSAGNE ( $\left.{ }^{1}\right)$}

Station de Recherches avicoles,

Centre national de Recherches zootechniques, Jouy-en-Josas (S \& O).

I a poule pond un ou plusieurs oufs en suivant, s'arrête généralement un jour, puis pond à nouveau un ou plusieurs œufs. Nous appelons pause le jour d'arrêt, et série la succession ininterrompue d'œufs comprise entre deux pauses.

I es premiers, BaStian et Zarrow, en I955, ont recherché si le poids du jaune était ou non constant en fonction de la place de l'œuf dans la série. I es mesures effectuées par ces deux auteurs sur le jaune à l'état frais ont mis en évidence une diminution de son poids du premier au dernier œuf dans les séries de 2 et 3 œufs.

De plus, les expériences d'ovulations prématurées effectuées à l'aide d'injection de $\mathrm{I}_{1} \mathrm{H}$ autorisent les conclusions suivantes :

- L'aptitude du follicule à se rompre est fonction de la dose; c'est-à-dire que l'ovulation provoquée d'un ovocyte est obtenue d'autant plus tôt par rapport à l'heure d'ovulation prévue que la dose de LH injectée est plus forte (BASTIAN et Zarrow I955).

- L'induction d'ovulations prématurées, à l'aide d'une injection de $\mathrm{I}, \mathrm{H}$, nécessite des doses moins importantes pour le premier follicule de la série que pour les suivants, lorsque le traitement est effectué pour chaque follicule à des temps égaux avant l'heure d'ovulation prévue (Fraps et Dury I942; Bastian et Zarrow 1955).

(1) Avec la collaboration technique de M11es (iate et Platiau. 
- Les ovocytes ainsi détachés prématurément de la grappe ovarienne renferment des quantités de vitellus d'autant moindres que la prématurité est forte (NEHER et Fraps I950).

Ces différents résultats nous ont amené à formuler 1'hypothèse selon laquelle, dans les conditions normales de ponte, le poids de vitellus renfermé dans l'ovule pouvait être un témoin de la durée d'évolution de la phase de grand accroissement du follicule qui le renfermait et que cette durée était inégale suivant la place de l'ovule dans la série. C'est pour vérifier cette idée et apporter quelques faits nouveaux susceptibles de mieux éclairer le déterminisme de la formation des séries, que nous avons entrepris l'étude comparée des réserves vitellines et de la durée de la phase de grand accroissement de l'ovocyte qui les renferme en fonction de sa place dans la série. Nous avons en outre étudié ces deux variables en fonction de l'âge de la poule afin de confirmer un travail préliminaire déjà effectué sur ce sujet (LACASSAGNE I957).

\section{Animaux}

Cette étude a été effectuée sur 9 animaux répartis en deux lots d'âge différent : le premier lot comprenait les poules $\mathrm{n}^{0} 5,6,7,8$, Io, le second les animaux $n^{0} 26,28,30,34$. Le contrôle des poules $n^{0} 5,6,7,8$, Io a commencé le II avril I957 et s'est terminé le 2 janvier I958. Les animaux avaient I I mois en début d'expérience et I9,5 mois en fin d'expérience.

Les poules $n^{\circ} 26,28,30,34$, plus jeunes, ont été suivies du 24 décembre 1957 au 3 juin 1958 . Dans ce cas l'âge était de 8 mois en début d'expérience et de $\mathrm{I} 3$ mois à la fin.

Les deux lots ont reçu durant toute cette période un éclairement constant de $\mathrm{I}_{3}$ heures par jour. Seul le premier groupe fut partiellement conditionné en température qui est demeurée, durant toute l'expérience, égale ou supérieure à $\mathrm{I} 2^{\circ} \mathrm{C}$.

\section{Méthode}

La durée de la phase de grand accroissement de l'ovocyte a été étudiée à l'aide d'une méthode par ingestion de colorants liposolubles. Ces colorants en se déposant dans le vitellus en zones concentriques permettent une fois l'œuf pondu de reconstituer la durée d'évolution du follicule au cours de l'accumulation de ses réserves (LACASSAGNe 1957). Cette technique est bien moins précise quant à l'heure de dépôt du colorant que celle employée par WARREN et CONRAD I939, mais il nous a semblé hasardeux d'effectuer toutes les 48 heures des injections intraveineuses de colorant à un même animal durant plus de 7 mois étant donné la difficulté de cette opération chez la poule.

Nous avons pris comme début de la phase de grand accroissement 
du follicule le premier dépôt de colorant dont 1e diamètre était égal ou supérieur à $7 \mathrm{~mm}$ car au-dessous de cette limite les mesures se sont avérées assez imprécises. RoMANOFF I93I et RIDDLE I9I6 font débuter cette phase de formation de l'ovocyte à un diamètre égal à $6 \mathrm{~mm}$. Pour tenir compte de ce fait nous avons majoré toutes les durées d'évolution de 12 heures.

Les colorants étant distribués toutes les 48 heures, les ovocytes à leur premier marquage avaient le plus souvent des diamètres compris entre 7 et $\mathrm{I}_{3} \mathrm{~mm}$. Conscients de l'erreur que pouvait introduire l'inégalité des premiers diamètres pris en considération, nous avons effectué les calculs d'une part sur les données brutes ainsi obtenues et d'autre part sur des données corrigées calculées en tenant compte de ce diamètre. Les corrections, en fonction de la dimension du premier dépôt de colorant d'un diamètre supérieur à $7 \mathrm{~mm}$, ont été effectuées selon le tableau suivant :

\section{TABLEAU I}

Diamètre du follicule au moment de son premier marquage (en $\mathrm{mm}$ ). Correction effectuée sur les don, nées brutes en jours ...........

$\begin{array}{ccccccccc}7 & 8 & 9 & \text { I0 } & \text { II } & \text { I2 } & \text { I3 } & \text { I4 } & \text { I5 } \\ 0 & 0 & +0,5 & +0,5+\mathrm{I} & +\mathrm{I} & +\mathrm{I}, 5 & +\mathrm{I}, 5+2\end{array}$

Cette table a été dressée à partir des données établies par WARREN et CONRAD en I939 sur la vitesse de croissance de l'ovocyte dans les premiers stades de la phase de grand accroissement. Nous verrons que les chiffres ainsi obtenus donnent des moyennes légèrement supérieures mais comparables, pour un grand nombre d'observations, à celles résultant des données brutes qui s'avèrent ainsi suffisantes en première approximation.

Dans l'un et l'autre cas, nous avons fait finir la phase de grand accroissement à o heure le jour de l'ovulation déterminé à partir du jour de ponte comme nous y autorisent les travaux de WARREN et SCOTT (I934, I935), Philips et WARREN (I937), Fraps (I955). Cette façon de procéder introduit une nouvelle erreur par défaut car, étant donné les conditions d'éclairement au cours de l'expérience ( 5 heures, I 8 heures) les ovulations avaient lieu après minuit. Nous reviendrons un peu plus loin sur l'estimation de cette erreur en fonction de la place de l'ovocyte dans la série. Chaque ovule a été examiné afin de vérifier la concordance de l'heure d'ovulation ainsi obtenue avec celle découlant du dépôt de colorant. La confrontation de ces deux données nous a montré que certains ovocytes, une fois leur croissance terminée, semblaient attendre quelquefois plus de 24 heures la déhiscence du follicule. Dans ces cas nous avons pris comme valeur de la durée d'évolution, le nombre de jour séparant le début de la phase de grand accroissement de la fin du dépôt de vitellus à l'intérieur du follicule sans égard à son heure de déhiscence. 


\section{Variation de la durée d'évolution de la phase de grand accroissement de l'ovocyte en fonction du vieillissement de la poule}

Nous avons divisé pour chaque animal, la durée de l'expérience en deux parties aussi équivalentes que possible en ce qui concerne le nombre d'œufs pondus, mais souvent très différentes quant au nombre de jours considérés, car l'intensité de ponte fut généralement plus faible dans la deuxième période d'observation que durant la première. Au tableau II figurent en regard de chacune de ces périodes les durées moyennes de croissance de l'ovocyte calculées à l'aide des données brutes telles que nous les avons définies au paragraphe précédent. Ces chiffres suffisent ici, car la valeur moyenne du premier diamètre pris en considération est identique pour chaque poule dans l'une et l'autre période. S'y trouvent également les poids moyens des réserves vitellines des ovocytes correspondants exprimés en extrait sec (vide $7^{2} \mathrm{~mm} \mathrm{Hg}, \mathrm{t}=70^{\circ} \mathrm{C}$. durant 48 heures).

Nous constatons dans tous les cas sauf un, celui de la poule 28 , une augmentation significative de la durée d'évolution de la phase de grand accroissement au cours de la deuxième période. Cette augmentation est corrélative d'un abaissement de l'intensité de ponte que l'on tienne compte ou non des pauses supérieures à 24 heures intervenues en cours de contrôle. Ces résultats sont en accord avec ceux déjà publiés (LACASSAGNE I957). L'exception de la poule 28 peut s'expliquer du fait que cet animal a présenté une plus forte intensité durant la deuxième phase de l'expérience que durant la première.

Ce tableau fait également ressortir une augmentation des réserves vitellines au cours du vieillissement de la poule. Cette différence s'inscrit dans l'évolution normale du poids de l'ovule au cours de la vie de l'animal (Romanoff I949). Le comportement différent de la poule 5 tient vraisemblablement à ce que cet animal a souffert d'une patte accidentellement meurtrie durant la deuxième période et a présenté de ce fait un amaigrissement d'environ $900 \mathrm{~g}$.

Il était intéressant de comparer la quantité moyenne de vitellus déposé par jour dans les ovocytes durant la première et la deuxième période de contrôle, c'est ce que nous avons exprimé dans la dernière colonne du tableau II. Les poules s'y trouvent divisées en deux groupes qui correspondent aux deux séries d'animaux employées dans cette étude. L,es poules $5,6,7,8$, Io présentent entre le onzième et le dix-huitième mois de leur existence un ralentissement du dépôt quotidien de vitellus dans le follicule. Il n'en est pas de même des animaux 28,30, 34 qui manifestent au contraire une augmentation du dépôt quotidien des réserves vitellines. Comme dans les deux cas nous assistons à une augmentation 


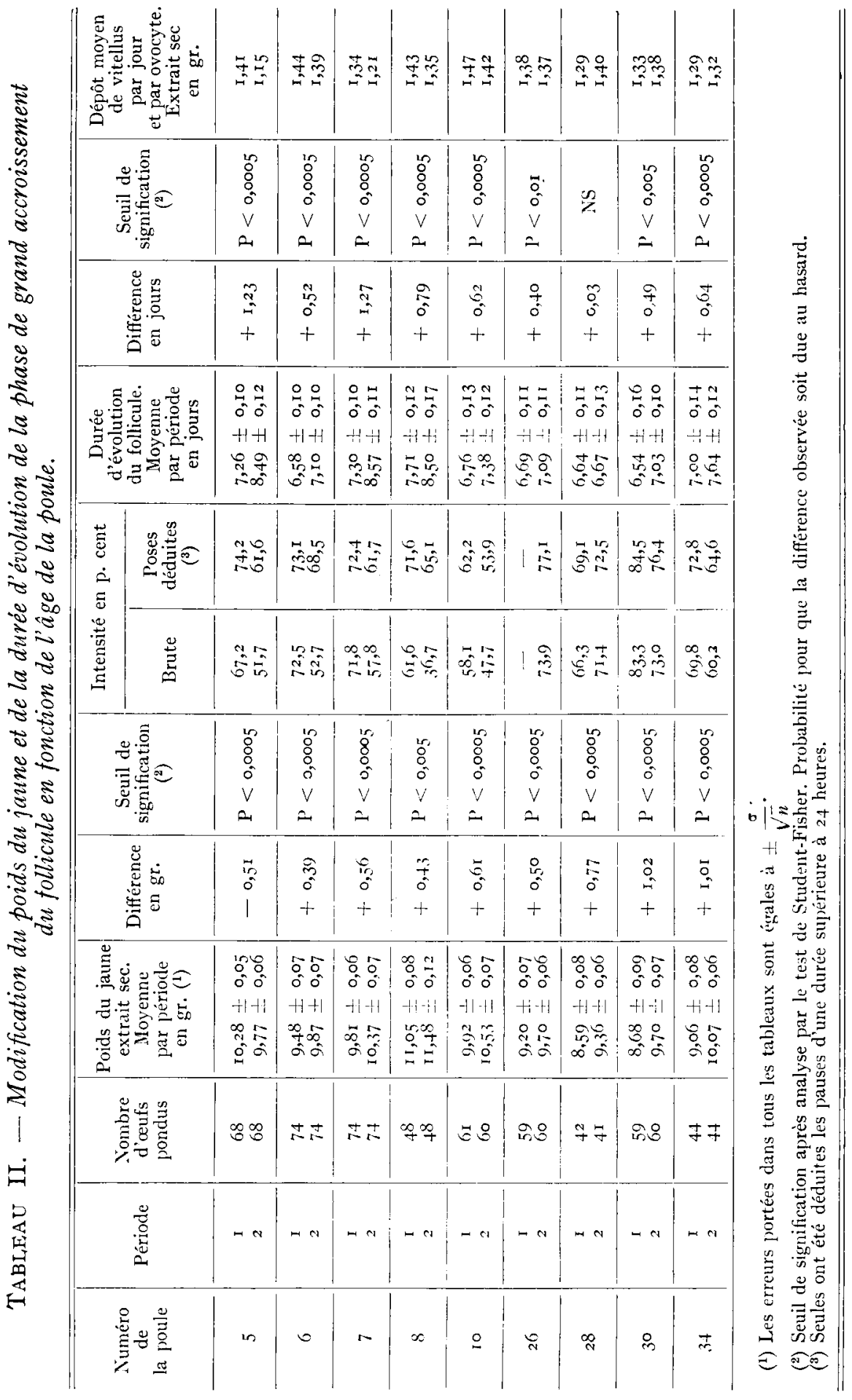


du poids de l'ovule (poule 5 excepté) et de la durée d'évolution du follicule, ces résultats montrent que chez les poules no $28,30,34$ l'augmentation de poids du vitellus en fonction du temps fut plus rapide que l'accroissement de la durée d'évolution ; inversement pour les animaux $n^{\circ} 5$, $6,7,8$, Io. I a poule 26 peut être classée à part étant donné la faible différence observée entre les deux périodes. Cette inégalité de comportement entre les deux groupes s'explique du fait que les poules $28,30,34$, plus jeunes, se trouvaient au moment de l'expérience dans une phase plus rapide d'accroissement du poids du jaune. Nous savons en effet depuis CuRTrs I9I4 que ce poids évolue rapidement en début de ponte puis de plus en plus lentement au fur et à mesure que la poule avance dans sa première année de production.

\section{Poids de l'ovule en fonction de sa place dans la série}

BASTIAN et ZARROW en I955 ont déjà essayé de mettre en évidence une relation entre le poids de l'ovule et sa place dans la série. Nous avons repris cette étude non plus sur le poids total du jaune à l'état frais, comme l'avaient fait ces deux auteurs, mais sur l'extrait sec afin d'éliminer une éventuelle variation de la teneur en eau du vitellus. Nos résultats reportés au tableau III confirment l'existence d'une diminution significative du poids du jaune entre le premier et le deuxiène œuf dans les séries de deux. Cette différence n'est pas significative dans les séries de trois où cependant nous observons une diminution très nette des réserves vitellines entre le deuxième et le troisième œuf.

\section{TABLEAU III}

Poids moyen des réserves vitellines de chaque ovule en fonction de la longueur de la série et de sa place dans cette série. (exprimé ell extrait sec)

\begin{tabular}{|c|c|c|c|c|c|}
\hline$\underset{\text { série }}{\text { Longueur de la }}$ & $\begin{array}{c}\text { Numéro d'ordre } \\
\text { dans la série }\end{array}$ & $\begin{array}{l}\text { Nombres de } \\
\text { stiries }\end{array}$ & $\begin{array}{c}\text { Poids moyen } \\
\text { de vitellus en gr. }\end{array}$ & $\begin{array}{l}\text { Différencess } \\
\text { en gr. }\end{array}$ & $\begin{array}{c}\text { Seuil de } \\
\text { signifination }\left({ }^{1}\right)\end{array}$ \\
\hline 2 œufs & $\begin{array}{ll}\mathrm{C} & \mathrm{I} \\
\mathrm{C} & 2\end{array}$ & 106 & $\begin{array}{r}10,120=0,071 \\
9,88+\cdots 0,066\end{array}$ & $-0,236$ & $\mathbf{P}<0,0005$ \\
\hline 3 œufs & $\begin{array}{l}\mathrm{C} \\
\mathrm{C} \\
\mathrm{C} \\
\mathrm{C}\end{array}$ & $\sigma_{3}$ & $\begin{array}{l}0,879 \div 0,099 \\
0,850 \div 0,097 \\
9,589 \div 0,098\end{array}$ & $\begin{array}{r}-0,029 \\
-0,261\end{array}$ & $\mathrm{P}<\mathrm{OS}$ \\
\hline $4 œ u f s$ & $\begin{array}{l}\mathrm{C} \\
\mathrm{C} \\
\mathrm{C} \\
\mathrm{C} \\
4\end{array}$ & 18 & 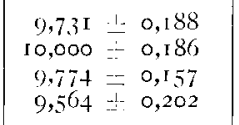 & $\begin{array}{l}+0,269 \\
-0,226 \\
-0,210\end{array}$ & 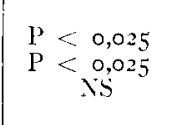 \\
\hline 5 oufs & $\begin{array}{ll}\text { C I } \\
\text { C } 2 \\
\text { C } 3 \\
\text { C } 4 \\
\text { C } 5\end{array}$ & It & 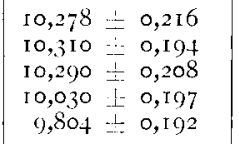 & $\begin{array}{l}+0,0,32 \\
-0,020 \\
-0,260 \\
-0,226\end{array}$ & $\begin{array}{c}\mathrm{NS} \\
\mathrm{P}<0,0005 \\
\mathrm{P}<0,0005\end{array}$ \\
\hline
\end{tabular}

(1) Seuil de sionification après analyse par la míthode des couples. 
I,es moyennes se rapportant aux séries de 4 et 5 œufs, bien que calculées à partir d'un nombre restreint de mesures, font également ressortir une chute nette du poids de vitellus en fin de série. Ent début de série au contraire le poids des réserves vitellines croît entre le ${ }^{\text {er }}$ et le $2^{\mathrm{e}}$ ovule pour descendre ensuite. Il semble donc que la vitellogenèse se fasse par à coups et soit plus importante en début qu'en fin de série. Ceci d'autant plus que le vitellus produit en 24 heures se trouve réparti sur l'ensemble des ovocytes en évolution sur la grappe ovarienne.

Cependant les valeurs du tableau III ne sont que des moyennes ne rendant pas exactement compte des résultats. In effet un nombre assez important d'œufs échappe à cette règle générale ; ainsi sur Io6 séries de deux aeufs nous en avons trouvé 34 dont les réserves vitellines étaient plus importantes dans le dernier ovule $\mathrm{C}_{2}$ que dans le premier $\mathrm{C}_{1}$. Un test par la méthode de $\chi^{2}$ effectué sur l'hypothèse d'une probabilité 5o pour Ioo d'avoir un poids égal pour $\mathrm{C}_{1}$ et $\mathrm{C}_{2}$ nous montre qu'il existe une différence significative $(\mathrm{P}<0,025)$ entre ces 34 exceptions et les 72 séries qui suivent la loi générale telle qu'elle apparaît au tableau III. De même sur 63 séries de trois œufs nous n'en trouvons que 33 où $\mathrm{C}_{2}$ est plus léger que $\mathrm{C}_{1}$ et 49 où $\mathrm{C}_{3}$ est plus léger que $\mathrm{C}_{2}$.

Pour les séries de 2 et 3 œufs nos résultats sont en accord avec ceux de BASTIAN et ZARROW ce qui tend à prouver que la teneur en eau du vitellus est relativement constante quelle que soit la place de l'ovule dans la série. Pour les séries plus longues la similitude s'arrête au deuxième ovule mais l'échantillonnage est faible dans l'un et l'autre cas (voir tableau IV).

TABLEAU IV

Relation entre le poids du jaune à l'état frais

et la position de l'ovmle dans la série (Bastian et Zarrow I955)

\begin{tabular}{|c|c|c|c|c|c|c|c|c|}
\hline \multirow{2}{*}{ Longrueur de la série } & \multirow{2}{*}{$\begin{array}{l}\text { Nombre } \\
\text { de séries }\end{array}$} & \multicolumn{6}{|c|}{$\begin{array}{c}\text { Poids moven du jaune en fonction du mumiro } \\
\text { d'ordre dans la sćrie (en g) }\end{array}$} & \multirow{2}{*}{$\begin{array}{c}\text { Chute } \\
\text { de poids } \\
\text { par jaune }\end{array}$} \\
\hline & & $\mathrm{C}_{1}$ & $\mathrm{C}_{2}$ & $\mathrm{C}_{3}$ & $\mathrm{C}_{4}$ & $\mathrm{C}_{5}$ & $C_{i s}$ & \\
\hline 2 oufs ... & $9^{6}$ & $I 7,5,5$ & $\mathrm{I} 7,3 \mathrm{I}$ & & & & & $0,2.4$ \\
\hline $3 œ u f s$ & 26 & $I 7,30$ & 17,65 & $1,-2 \mathrm{I}$ & & & & $0,3+$ \\
\hline 4 oufs & 9) & 57,13 & 17,60 & 17,72 & 17,10 & & & $-0,09$ \\
\hline 5 oufs $\ldots \ldots \ldots$ & 2 & $\mathrm{I} 7,1,0$ & 17,70 & 17,90 & $1,7,55$ & 17,60 & & 0,00 \\
\hline 6 oeufs . . . . . . . . . & 2 & $16,3.5$ & 16,75 & $I 7,30$ & 17,30 & Is, 20 & 18.70 & $-0,4 \mathrm{I}$ \\
\hline
\end{tabular}

\section{Durée d'évolution de la phase de grand accroissement de l'ovoeyte en fonction de sa place dans la série et de la longueur de cette série.}

I,es données brutes autant que les données corrigées (tableau V) font ressortir une diminution de la durée d'évolution de la phase de grand accroissement du follicule au fur et à mesure que 1'on va vers la fin de la 


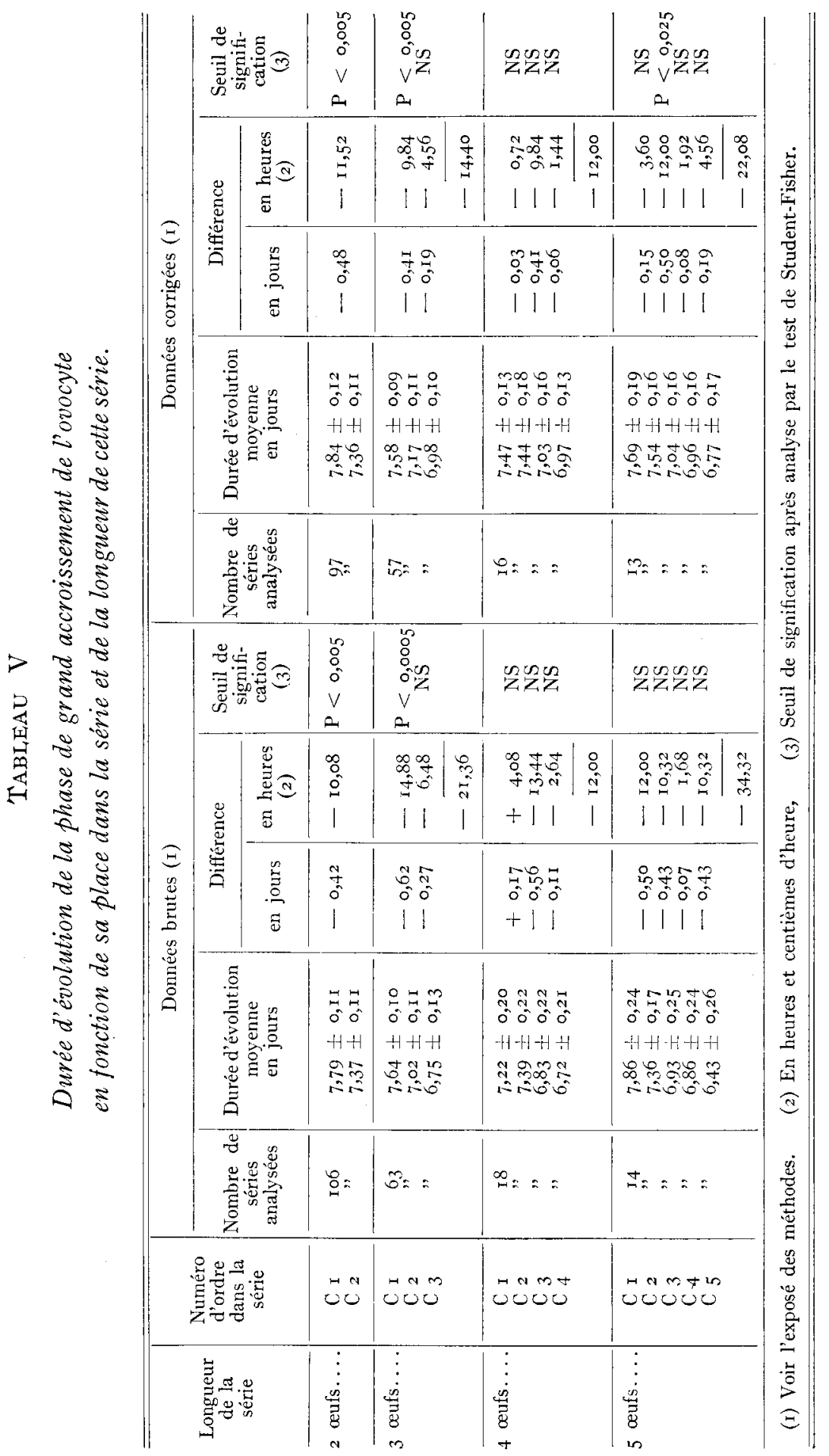


série. Ce tableau ne mentionne le seuil de signification que pour les follicules immédiatement voisins. En fait dans les séries de 4 œufs si la différence de la durée d'évolution entre $C_{1}$ et $C_{2}$ n'est pas significative, celle entre $C_{1}$ et $C_{3}$ et a plus forte raison celle entre $C_{1}$ et $C_{4}$ le sont. De même entre $C_{2}$ et $C_{4}$. Dans les séries de 5 œufs nous avons également établi que les différences entre $C_{1}$ et $C_{3}, C_{1}$ et $C_{4}, C_{1}$ et $C_{5}, C_{2}$ et $C_{4}, C_{2}$ et $C_{5}$ sont significatives (tableau VI). Mais les différences réelles entre ovocytes sont en réalité moins importantes qu'elles ne paraissent dans ces tableaux. En effet comme nous l'avons fait remarquer dans l'exposé des méthodes ces données sont entachées d'erreurs qui proviennent de ce que nous avons arrêté la phase de grand accroissement du follicule à o heure la veille du jour de ponte, alors que les ovulations ont lieu quelques heures après minuit. En nous rapportant aux chiffres publiés par FRAPS 1955 , il est possible d'évaluer l'erreur obsolue par défaut faite sur $\mathrm{C}_{1}$ dans les conditions de l'expérience à environ 4 heures 30 minutes. L'erreur absolue commise sur les autres follicules de la série est supérieure à 4 heures 30 puisque l'heure d'ovulation se retarde d'un jour sur l'autre au cours d'une même série. Pour l'interprétation des résultats, il est important de connaitre non l'erreur absolue mais l'erreur relative commise sur les différents ovules d'une même série. Nous emprunterons pour cette estimation les chiffres de I'RaPs (I955) (tableau VII) déduits des heures d'oviposition en fonction de la longueur de la série.

\section{TABIHAL VI}

Différence entre les duvées moyennes d'évolution de deux ovocytes non consécutifs dans les séries de 4 et 5 cufs.

\begin{tabular}{|c|c|c|c|c|c|}
\hline \multirow{2}{*}{$\begin{array}{l}\text { Longueur } \\
\text { dé la } \\
\text { série }\end{array}$} & \multicolumn{3}{|c|}{ Données brutes } & \multicolumn{2}{|c|}{ Domnées corrigées } \\
\hline & $\begin{array}{l}\text { Numéro d'ordre } \\
\text { dans la série }\end{array}$ & \begin{tabular}{|} 
Durééevolution \\
Différence \\
en jours
\end{tabular} & Signification & $\begin{array}{c}\text { Duréc évolution } \\
\text { Différence } \\
\text { en jours }\end{array}$ & Signification \\
\hline 4 œufs ... & $\begin{array}{l}\text { Entre } C_{1} \text { et } C_{3} \\
\text { Entre } C_{1} \text { et } C_{4} \\
\text { Entre } C_{2} \text { et } C_{4}\end{array}$ & $\begin{array}{r}-0,30 \\
-0,50 \\
-0,07\end{array}$ & $\begin{array}{l}\mathrm{NS} \\
\mathrm{NS} \\
\mathrm{r} 0,025\end{array}$ & $\begin{array}{l}-0,44 \\
\cdots \quad 0,50 \\
-0,47\end{array}$ & $\begin{array}{l}\mathbf{P}<0,025 \\
P^{\prime}<0,01 \\
P<0,025\end{array}$ \\
\hline 5 oufs & $\begin{array}{l}\text { Entre } C_{1} \text { et } C_{3} \\
\text { Entre } C_{1} \text { et } C_{4} \\
\text { Entre } C_{1} \text { et } C_{5} \\
\text { Entre } C_{2} \text { et } C_{1} \\
\text { Entre } C_{2} \text { et } C_{5}\end{array}$ & $\begin{array}{l}-0,9,3 \\
-\quad 1,00 \\
-\quad 1,43 \\
-0,50 \\
-0,0,3\end{array}$ & $\begin{array}{l}\mathrm{P}<0,01 \\
\mathrm{P}<0,005 \\
\mathrm{P}<0,0005 \\
\mathrm{NS} \\
\mathrm{P}<0,01\end{array}$ & $\begin{array}{l}-0,6,5 \\
-\quad 0,7,3 \\
-0,92 \\
-0,78 \\
-0,77\end{array}$ & $\begin{array}{l}P^{\prime}<0,01 \\
P^{\prime}<0,005 \\
P^{\prime}<0,0001 \\
P^{\prime}<0,025 \\
P^{\prime}<0,005\end{array}$ \\
\hline
\end{tabular}

Ces données sont en accord avec nos observations et peuvent également se déduire de travaux antérieurs tels que ceux de Atwood I929, HAYS I936, HEYWANG I938, BERG I945.

$I_{\downarrow}$ a diminution de la durée d'évolution entre le premier et le dernier ovocyte de la série, dans le cas de notre expérience, est bien supérieure 
au retard total de l'heure d'ovulation par série tel qu'il ressort du tableau de FraPs.

TABLEAU VII

Retard de l'heure d'ovulation de Leghorns blanches en fonction de la place de l'ovule dans la série (exprimé en heure et centièmes d'heure) (FRAPS I955).

\begin{tabular}{|c|c|c|c|c|c|}
\hline \multirow{2}{*}{$\begin{array}{l}\text { Longueur de la } \\
\text { série }\end{array}$} & \multicolumn{4}{|c|}{ Retard entre les différents ovules de la séric } & \multirow{2}{*}{$\begin{array}{l}\text { Retard total } \\
\text { par séries }\end{array}$} \\
\hline & entre $C_{1}$ et $C_{2}$ & entre $\mathrm{C}_{2}$ et $\mathrm{C}_{3}$ & entro $\mathrm{C}_{3}$ et $\mathrm{C}_{4}$ & entre $\mathrm{C}_{4}$ et $\mathrm{C}_{5}$ & \\
\hline 2 & $4,5.3$ & & & & $4,5,3$ \\
\hline 3 & 3,97 & 2,72 & & & 6,68 \\
\hline 4 & 3,47 & 1,98 & $1,0,3$ & & $7,3^{8}$ \\
\hline 5 & $3, I_{3}$ & I,, 63 & $\mathrm{I}, 77$ & 1,52 & 7,75 \\
\hline
\end{tabular}

Nous pensons donc être en mesure de conclure, malgré toutes les réserves antérieures, à une diminution réelle de la durée d'évolution du follicule au cours de la série. Ceci confirme 1'hypothèse d'une "maturité " moindre des ovocytes en fin de série qui se manifeste également par un poids inférieur des réserves vitellines. I,e follicule peut donc se rompre avec des réserves différentes mais vraisemblablement au-dessus d'un certain seuil représenté par le poids de vitellus du dernier ouf de la série, sans préjuger du rôle joué par ce vitellus dans la déhiscence du follicule. A l'intérieur d'une même série le temps nécessaire à l'accumulation de réserves identiques dans deux follicules successifs apparaît donc comme relativement constant et généralement supérieur à l'intervalle séparant deux ovulations. Nous ne devons pas oublier cependant qu'un certain pourcentage de séries échappe à cette règle. Dans ce dernier cas on peut penser que l'ovogenèse est plus rapide et l'intervalle séparant la matura tion de deux follicules plus court.

Il eut été intéressant de comparer les follicules $C_{1}$ des séries de deux œufs aux follicules $C_{1}$ des séries de 3,4 et 5 œufs. Mais les chiffres reportés au tableau $\mathrm{V}$ ne nous autorisaient pas à effectuer cette étude ; 1'intensité de ponte ayant varié au cours de l'expérience nous aurions retrouvé les conclusions du tableau II.

Signalons enfin que de nombreux ovocytes $C_{1}$ une fois leur croissance terminée semblent attendre sur l'ovaire, souvent durant 24 heures, la déhiscence du follicule sans que nous puissions affirmer dans tous les cas qu'il ne s'agit pas simplement d'une oviposition retardée.

\section{RÉSUMÉ}

Nous avons étudié, comparativement, la durée de la phase de grand accroissement et la quantité de réserves vitellines de chaque ovocyte chez la poule domestique en fonction de son âge et de la place du folli- 
cule dans la série. Cette étude nous a amené aux conslusions suivantes :

- L'abaissement de l'intensité de ponte, corrélative de l'augmentation de poids des ovocytes au cours de la première année de production, est dû à une évolution plus lente du follicule généralement jointe à une vitellogénèse diminuée.

- La durée d'évolution de la phase de grand accroissement du premier follicule de la série est supérieure à celle des autres follicules de cette série. La durée d'évolution des ovocytes suivants diminue au fur et à mesure que l'on se déplace vers la fin de la série.

- La quantité de réserves vitellines par ovule suit une évolution analogue dans les séries de 2 œufs. Mais dans les séries de 3,4 et 5 œufs ces réserves croissent ou demeurent égales du $I^{\mathrm{er}}$ au $2^{\mathrm{e}}$ ovule pour ensuite diminuer.

- Ces résultats mettent eil évidence une corrélation poids de vitellus - durée d'évolution.

\section{SUMMARY}

A comparative study has been made between the length of the rapid growth phase of each ovocyte of the domestic fowl and the vitelline reservesl with the relationship to the age of the animal and the position of the follicle in the clutch.

The following conclusions may be drawn :

- The lowering in intensity of laying during the first year of production correlating with an increase in weight of each of the ovocytes, is due to a slower evolution of the follicle which is generally combined with a reduced vitellogenesis.

- The length of evolution of the rapid growth phase of the first follicle of a clutch is longer than that of the subsequent follicles. Evolution of the other ovocytes becomes progressively shorter as the end of the clutch approaches.

- The decrease in amount of vitelline reserves per ovule in clutches of two eggs, occurs in a similar manner. But, in clutches of three for and five eggs, these reserves increase or remain the same from the first to the second ovule and then diminish.

- These results demonstrate a correlation between the weight of the vitellus and the length of its evolution.

\section{RÉFÉRENCES BIBLIOGRAPHIQUES}

ATwOod (H.). - Observations concerning the time factor in egg production Poultry Science, 8, I37-I40, I929.

Bastian (J. W.) and Zarrow (M. X.). - A New hypothesis for the asynchronous ovulatory cycle of the domestic hen (Gallus domesticus). Poultry Science, 34, $776-788$, 1955.

BERG ( $I_{1}$. R.). - The relationship of clutch position and time interval between eggs to eggshell quality. Poultry Science, 24, 555-563, I945.

Curtis (M. R.). - Arch. Entwicklungsmech. Organ., 39, 217-327, I9I4. 
Fraps (R. M.). - Progress in the physiology of farm animals. Butterworths scientific Publications. London, I955.

Fraps (R. M.), and DURY (A.). - Relative sensitivity to certain ovulation inducing agents of first and subsequent follicles of clutch sequences in the hen. Anat. Rec., 84, 453, I942.

Hays (F. A.). - The interval between eggs of Rhode Island Red Pullets. Journal of A gricultural Research, 52, 633-638, 1936.

Hrywang (B. W.). - The time factor in egg production. Poultry Science, $17,240-247,1938$.

I ACAssagre (L.). - Dynamique de l'ovogenèse. Contribution à l'étude de la phase de grand accroissement des follicules chez la pottle domestique. Annales de Zootechnie, 2, 85-03, I957.

NEHER (B. H.) and Frap's (R. M.). - The addition of eggs to the hen's clutch by repeated injections of ovulation-inducing hormones. Endocrinology, 46, $482-488$, I950.

Philims (R. E.) and WARREN (I). C.). - Observations concerning the mechanics of ovulation in the fowl. The Journal of Exp. Zool., 76, II7-I36, 1937.

RIDDLE (O.). - Studies on the physiology of reproduction in birds. I. - The occurrence and measurement of a sudden change in the rate of growth of avian ova. The Amer. J. Physiol., 41, 387-396, I9I6.

Romanoff (A. L.) and Romanoff (A. J.). - The avian egg. John Wiley and Sons, Inc., New York, I949.

ROMANOFF (A. L.). - Growth and chemical composition of ovum of fonctionning fowls ovary (gallus domesticus). The biochemical journal, 25, 994-996, I931.

WARren (D. C.) and Conrad (R. M.). - Growth of the hen's ovum. Journal of A gricultural Research, $\mathbf{5 8}, 875-893$, I939.

WARren (D. C.), and ScotT (H. M.). - Ovulation in the domestic hen. Science, 80, 46I-462, I934.

WARren (D. C.) and ScoTT (H. M.). - The time factor in egg formation. Poultry Science, 14, I95-207, I935.

Le Directeur-Gérant : B. LACL,AVIÈRE.

I.N.R.A.

BIBLIOTHEQUE UO \$58106e Bussière à Saint-Amand (Cher), France. - 5-4-1960.

DOMAINE OE CROUEL Léót légal: 2e trimestre 1960. No d'impression 826.

63039

Clermant-FD CEDEX? 